Review:

\title{
Progress in research on mixing techniques for transverse injection flow fields in supersonic crossflows
}

\author{
Wei HUANG ${ }^{\dagger}$, Li YAN \\ (Science and Technology on Scramjet Laboratory, National University of Defense Technology, Changsha 410073, China) \\ †E-mail: gladrain2001@163.com \\ Received Mar. 22, 2013; Revision accepted June 13, 2013; Crosschecked July 10, 2013
}

\begin{abstract}
The transverse injection flow field has an important impact on the flowpath design of scramjet engines. At present a combination of the transverse injection scheme and any other flame holder has been widely employed in hypersonic propulsion systems to promote the mixing process between the fuel and the supersonic freestream; combustion efficiency has been improved thereby, as well as engine thrust. Research on mixing techniques for the transverse injection flow field is summarized from four aspects, namely the jet-to-crossflow pressure ratio, the geometric configuration of the injection port, the number of injection ports, and the injection angle. In conclusion, urgent investigations of mixing techniques of the transverse injection flow field are proposed, especially data mining in the quantitative analytical results for transverse injection flow field, based on results from multi-objective design optimization theory.
\end{abstract}

Key words: Aerospace propulsion system, Transverse injection, Mixing, Supersonic crossflow doi:10.1631/jzus.A1300096 Document code: A CLC number: V43

\section{Introduction}

In the scramjet engine (Qin et al., 2012), it is necessary to promote the mixing process between the fuel and the supersonic crossflow before ignition, and thus enhance combustion efficiency (Wang et al., 2011a; Zhang et al., 2012). In the past decades, many fuel injection schemes have been proposed for scramjet engines (Takahashi et al., 2010b; Huang et al., 2011a; 2013c; Yang et al., 2011; Zhang et al., 2011), especially using a cantilevered ramp injector design as the inlet injector (Huang et al., 2013c) and the transverse injection scheme which has been widely employed in the scramjet combustor (Cecere et al., 2011). Seiner et al. (2001) reviewed the approaches to improving the mixing efficiency of fuel

\footnotetext{
${ }^{*}$ Project supported by the Science Foundation of National University of Defense Technology (No. JC11-01-02), and the Hunan Provincial Natural Science Foundation of China (No. 12jj4047)

(c) Zhejiang University and Springer-Verlag Berlin Heidelberg 2013
}

and the supersonic freestream in the scramjet engine, and pointed out that the ramp injector is one of the most promising candidates. However, this configuration does not have a smooth aerodynamic line and thus cannot give the strongest flame-holding ability. Its geometric configuration should therefore be modified, so that the generation of streamwise vortices is diminished and lower drag is achieved.

When fuel is injected into the flowpath from the forebody/inlet of the hypersonic vehicle, the length of the combustor is shortened, and the weight of the engine is lightened (Livingston et al., 2000; Guoskov et al., 2001; Huang et al., 2010; Wang and Sislian, 2010; Turner and Smart, 2010; Feng et al., 2011). The laser-induced plasma ignition characteristics of the inlet injection flow field in the scramjet engine were investigated by Brieschenk et al. (2013) using the planar laser-induced fluorescence technique, as shown in Fig. 1. This was the first laser spark study for inlet injection in hypersonic flow. This research demonstrated that the laser-induced plasma ignition 
technique could promote the formation of hydroxyl in hypersonic flow.

At the same time the transverse injection scheme can provide better fuel penetration performance, mixing performance, and heat release performance, than the parallel injection scheme when the incoming Mach number is much lower-but it will induce larger total pressure loss (Manna and Chakraborty, 2005). This implies that there must be a compromise between the objective functions of the supersonic transverse injection flow field, and that the flow field should be optimized by the multi-objective design optimization method. Thus, a quantitative relationship between the objective functions and the design variables, and a qualitative relationship between the objective functions can be obtained. In the current study, the transverse injection scheme means that the fuel is injected into supersonic crossflows transversely, and the parallel injection one means that the fuel is injected parallel to the supersonic freestream.

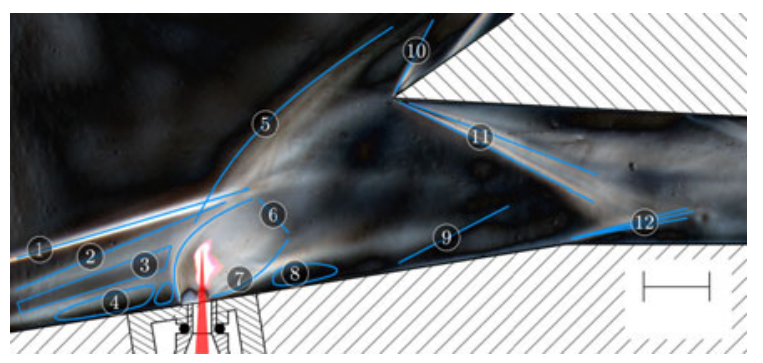

Fig. 1 Schlieren image of jet flow field in the forebody/inlet of the hypersonic vehicle (Brieschenk et al., 2013)

1: leading edge shock wave; 2: separation shock wave; 3 : separation boundary layer; 4: front recirculation region; 5: interactive bow shock wave; 6: mach disk; 7: jet incident/ barrel shock wave; 8: wake recirculation region; 9: recompression shock wave; 10: cowl shock wave; 11: cowl/ combustor shock waves; 12: rarefaction wave

However, the shock wave structure generated in an unsteady liquid fuel injection flow field, i.e. kerosene or diesel in this case, is more complicated (Xu et al., 2000) and includes four regimes in the atomization region, namely jet column zone, primary and secondary breakup zones, and droplets zone. This phenomenon is not observed in the transverse gaseous injection system, implying that the phase of the fuel has an impact on the structure of the transverse injection flow field. The hydrogen injection scheme is shown to be more efficient for increasing the cruising speed of a hypersonic vehicle (Wang et al., 2011b).
To the best of the authors' knowledge, Ogawa and Boyce (2012) first applied a surrogate-assisted evolutionary algorithm to optimize the fuel injection flow field in a scramjet engine, and the Pareto front has been obtained (Fig. 2). In Fig.2, $h_{\mathrm{p}}, \Delta p_{0}$, and $\eta_{\mathrm{m}}$ represent the fuel penetration height, the total pressure loss, and the mixing efficiency, respectively. The results obtained show that the injection angle and the aspect ratio of the injection port both have a large impact on the mixing of the fuel and the incoming flow, and the fuel penetration depth is determined by the injection angle and the distance between the injection ports. Further, the jet-to-crossflow pressure ratio is shown to be a key parameter governing the aerodynamic properties in the transverse injection flow field (Rizzetta, 1992; Beresh et al., 2006; Chen et al., 2011; Huang et al., 2012b).

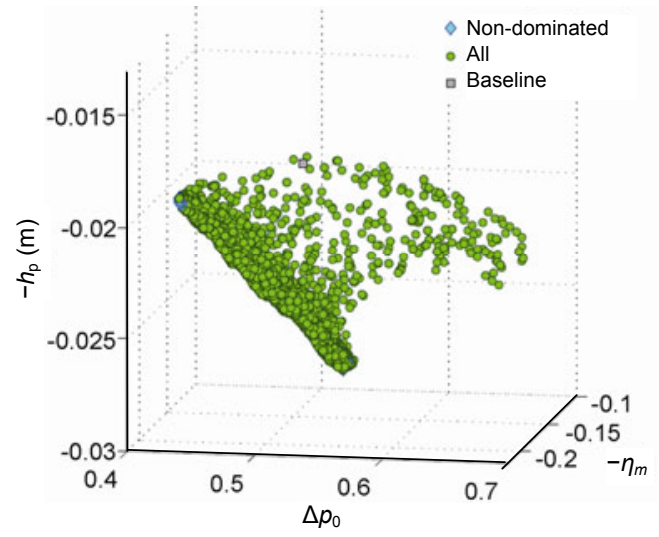

Fig. 2 Pareto front for the multi-objective design optimization of the transverse injection flow field (Ogawa and Boyce, 2012)

Therefore, in the current survey, research development on the mixing technique of the transverse injection flow field is summarized from four key aspects, namely the jet-to-crossflow pressure ratio, the geometric configuration of the injection port, the number of the injection ports and the injection angle. Finally, further urgent investigations which should be carried out on the transverse injection flow field are described.

\section{Effect of the jet-to-crossflow pressure ratio}

Aso et al. (1991) experimented on the influence of the jet-to-crossflow pressure ratio on the transverse injection flow field in supersonic flows, and found 
that the shapes of the bow shock wave, the Mach disk and the barrel shock wave are all enlarged with the increase of the jet-to-crossflow pressure ratio, and the separation point of the turbulent boundary layer moves upstream with the increase of the jet-tocrossflow pressure ratio. Accordingly, the separation region upstream of the injection port increases gradually. The fuel penetration depth and the jet-to -crossflow pressure ratio show a nearly linear relationship which is consistent with the conclusion obtained by Huang et al. (2012a), as shown in Fig. 3 .

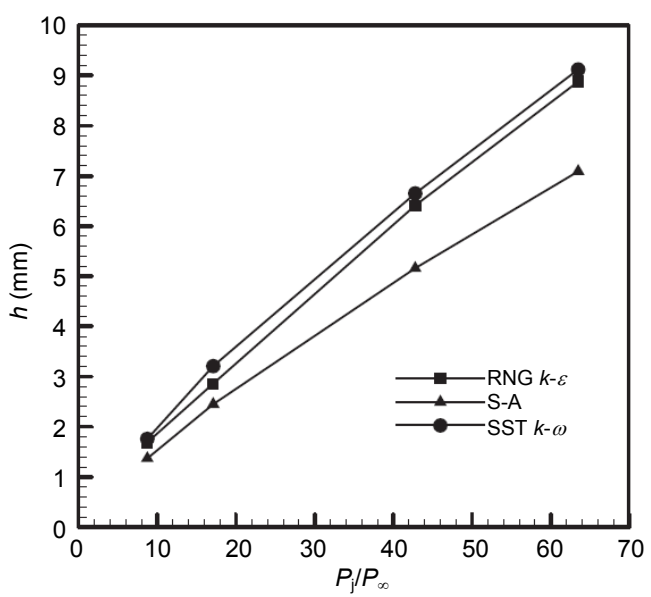

Fig. 3 Penetration depth versus jet-to-crossflow pressure ratio (Huang et al., 2012a)

$h$ and $P_{\mathrm{j}} / P_{\infty}$ represent the penetration depth and the jet-tocrossflow pressure ratio, respectively

A detached-eddy simulation (DES) model was employed by You et al. $(2012 ; 2013)$ to study a transverse injection flow field with a low jet-tocrossflow momentum flux ratio $(J=0.35)$ in the HyShot II scramjet system. They found that a combination of a detached normal shock wave and a 3D barrel shock wave is generated in the vicinity of the exit of the injection port when the jet-to-crossflow momentum flux ratio is low (Fig. 4). This is due to the simultaneous occurrence of the overexpansion and underexpansion conditions at the exit of the injection port. At the same time, the $\Omega$-shaped vortices, which were observed in previous experiments, were obtained, as shown in Fig. 5 (You et al., 2013). The formation of the $\Omega$-shaped vortices is due to the interaction between the unsteady Kelvin-Helmholtz $(\mathrm{K}-\mathrm{H})$ instability and the counter-rotating vortex pair (CVP) deformation. It is concluded that the $\Omega$-shaped vortices can provide better mixing performance than the CVP.

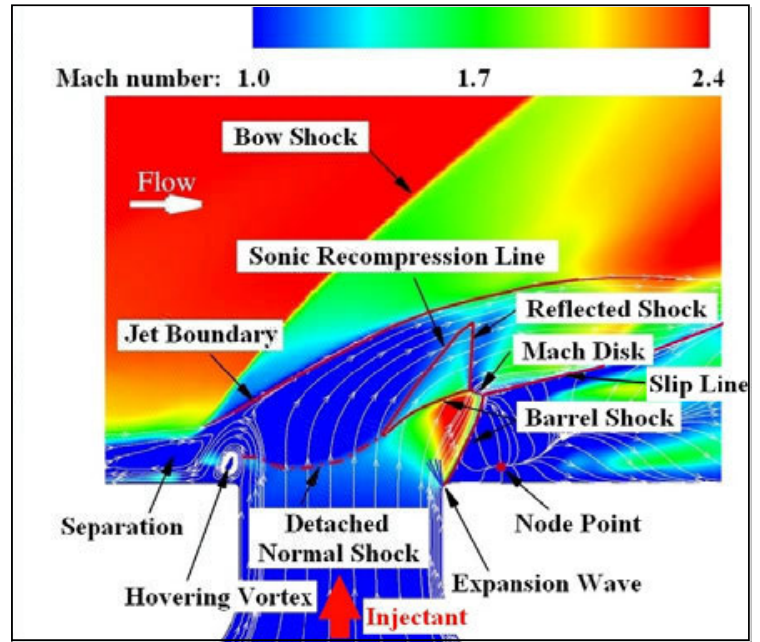

Fig. 4 Mach number contour in the symmetric plane (You et al., 2012)

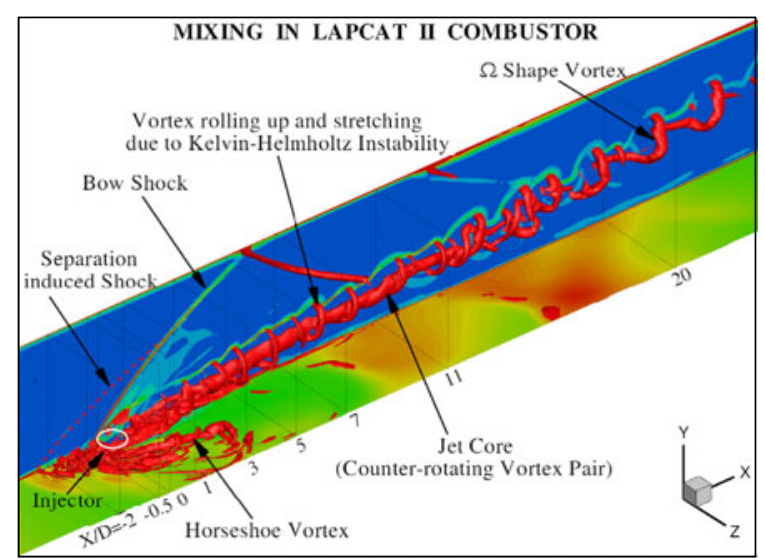

Fig. 5 3D vortex structure in the transverse injection flow field with low jet-to-crossflow momentum flux ratio (You et al., 2013)

\section{Effect of the geometric configuration of the injection port}

The flow field structure near the diamondshaped injection ports with different injection angles and two total pressures has been investigated experimentally by Bowersox et al. (2004). Their experimental approaches include surface oil flow visualization, shadowgraph photography, Mie scattering flow visualization, pressure-sensitive paint, and a pitot-cone five-hole pressure probe. Meanwhile, a $90^{\circ}$ circular injector, with the same exit port area and total pressures, was studied for comparative purposes. Finally, they obtained the penetration correlations for 
both the diamond and circular injectors, as shown in Fig. 6. It is concluded that the $90^{\circ}$ circular injector can generate the largest peak pressure for a given jet-to -crossflow pressure ratio, and its total pressure loss is the largest.

In Fig. $6, J_{\text {eff }}=J \sin \alpha=\rho_{\mathrm{j}} u_{\mathrm{j}}{ }^{2} \sin \alpha /\left(\rho_{\infty} u_{\infty}{ }^{2}\right)$, with $\alpha$ being the injection angle and $d$ the effective geometric diameter $(4.89 \mathrm{~mm}) . x$ and $y$ are the axial and transverse coordinates, respectively. $P_{\mathrm{t}}$ is the stagnation pressure for the supersonic freestream.

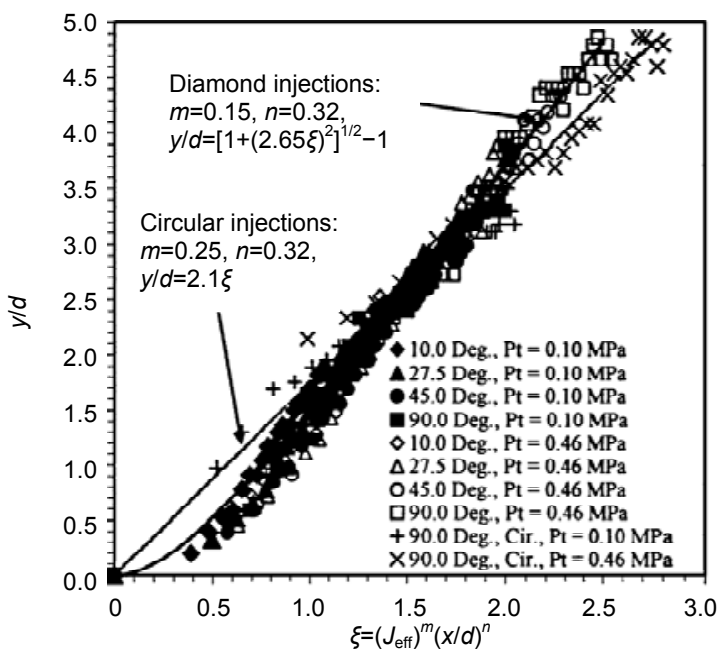

Fig. 6 Penetration depth correlation results (Bowersox $e$ al., 2004)

Srinivasan and Bowersox (2008) used both Reynolds-averaged Navier-Stokes (RANS) and DES models to study the transverse injection flow field structures with circular- and diamond-shaped injection ports in a Mach 5.0 freestream flow. They found that a lateral counter-rotating vortex pair (LCVP) is located just downstream of the barrel shock wave in the diamond-shaped injector flow field (Fig. 7). This structure can entrain fluid from the injection port, enabling the vortex pair to act as a potential flame holder to a certain extent. It can also reduce the heat transfer to the wall compared with the cavity flame holders and, at the same time, prolong the resident time compared with the streamline in the freestream. The configuration of the barrel shock wave has an important impact on the formation of the LCVP. The shock wave shape for the diamond-shaped injector facilitates the formation of the LCVP, and a pair of cone-shaped node structures is formed downstream of the LCVP (Fig. 7).

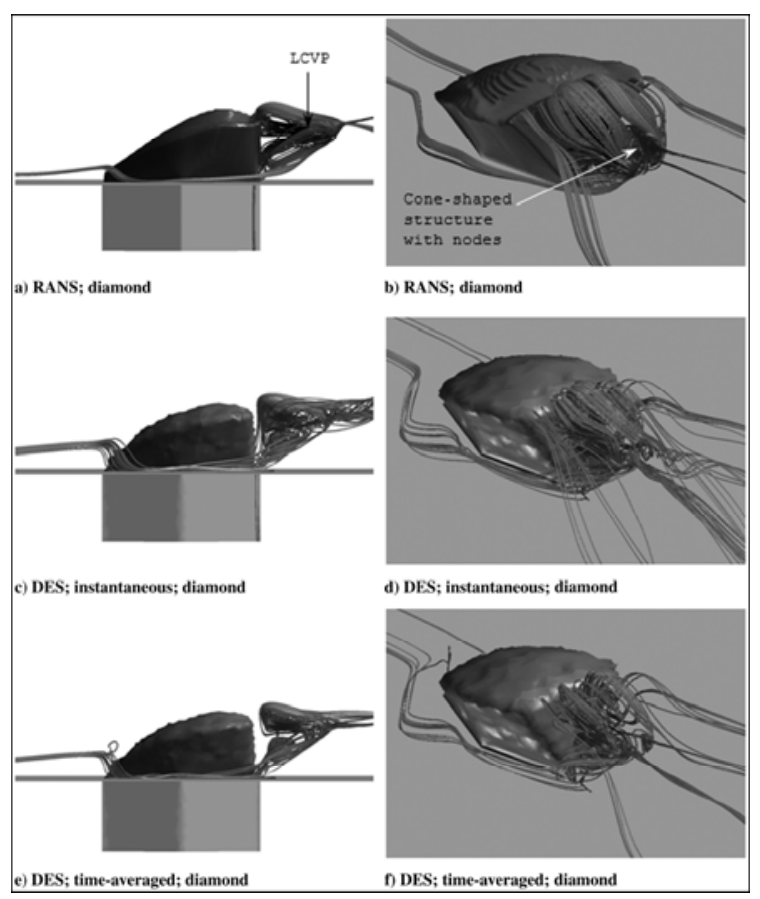

Fig. 7 Lateral counter-rotating vortex pair flow structure in the diamond-shaped injector configuration. Freestream flow is from left to right for left-column images and from top left to bottom right for right-column images (Srinivasan and Bowersox, 2008)

Based on the research carried out by Srinivasan and Bowersox (2008), the mixing and combustion flow fields using a diamond-shaped fuel injection port in a Mach 2.0 freestream were investigated experimentally and numerically by Kobayashi et al. (2010). Their aim was to visualize the structure of the LCVP and assess its reactivity. In the experiment, the NO-Planar Laser Induced Fluorescence (NO-PLIF) images captured the 3D structure of the LCVP for the first time; however, the combustion suitable for flame holding was not observed within the LCVP. This is due to the low pressure and temperature under the operating conditions, and the gas composition within the LCVP is very fuel-rich. Tomioka et al. (2011) investigated the combustion characteristics with diamond- and circular-shaped injection ports in a direct-connect supersonic combustor. They found that supersonic injection through the diamond-shaped injection port can result in a slightly larger pressure rise in the far field. However, the possibilities for ignition and mode transition (ramjet-to-scramjet mode combustion) are weaker due to less interaction 
between the injectant plume and the airstream near the injection wall.

At the same time, in the flow field with the diamond-shaped injection port, a pair of vortices is generated at the leading edge of the diamond-shaped injector exit, as shown in Fig. 8 (Srinivasan and Bowersox, 2008). The formation of this vortex pair is due to the shear on the injector exit plane along with the corner vorticity in the diamond-shaped injection port. This vortex pair can entrain fluid from the external region of the freestream boundary layer, as well as from the injection port. It can therefore improve the mixing efficiency between the freestream and the injector fluids. The circular-shaped injector can produce a somewhat similar flow field structure as well, which is similar to the horseshoe vortex.
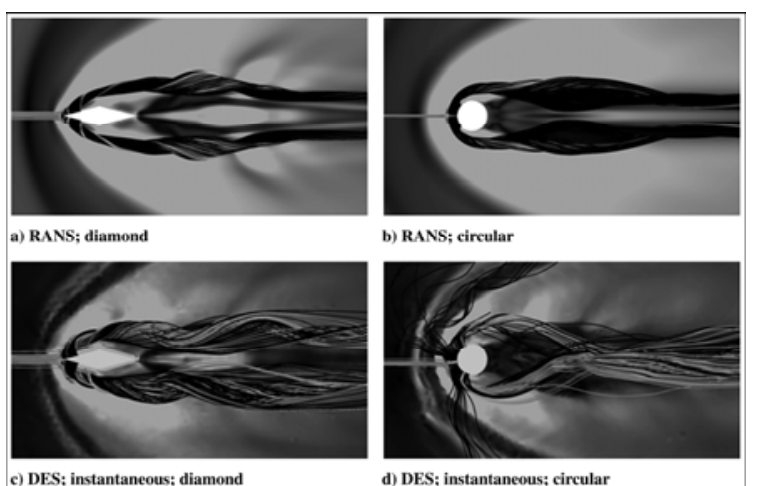

c) DFS; instantaneous; diamon

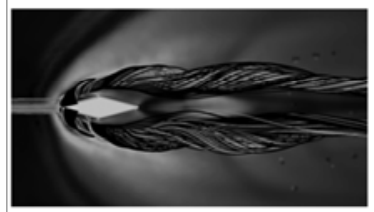

e) DES; time-averaged; diamond
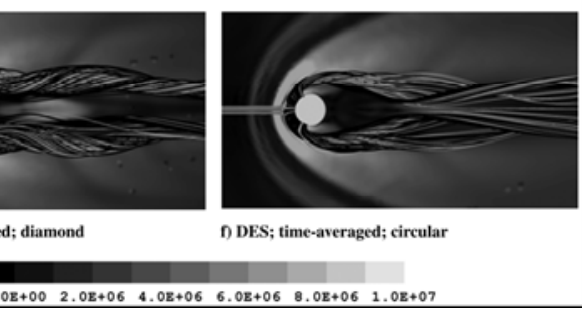

f) DES; time-averaged; circular

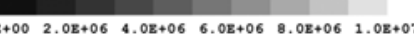

Fig. 8 Comparison of the leading-edge mixing vortex for circular- and diamond-shaped injection ports (Srinivasan and Bowersox, 2008)

\section{Effect of the number of injection ports}

The flow field of multi-port transverse injection into a supersonic crossflow is more complex than the single injection flow field (Fig. 9) (Lee, 2006a), and this is due to the strong interactions among the injection flows, various shock wave structures and vertical flows around the injection flows. An efficient injection system setup must include the selection of many parameters, i.e., the position of the injection port, the distribution of the mass flow rate and momentum flux, the injection angle, and the combination of the injection angles (Kovar and Schulein, 2006; Lee, 2006a; Li et al., 2012; Pudsey et al., 2012).

Fig. 9 shows the schematic view of the dual transverse injection flow field (Lee, 2006a). The supersonic freestream is blocked by the front jet, and the blockage effect has an important impact on the mixing process. There are many shock waves generated in this flow field: the 3D bow shock waves formed ahead of the front and rear jets, a separation shock wave generated by the interaction between the front bow shock wave and the boundary layer, and the Mach disks. A remarkable characteristic of the dual injection scheme is that the Mach disk of the rear jet is located at a higher position from the wall and is larger than the Mach disk of the front jet. At the same time, there are many vortical pairs formed along the jet direction, i.e., the horseshoe vortices, the separation bubble, and the recirculation wake flows. The evolution of the vortex pair is a very complex process involving 3D tilting and folding. The streamwise vortices roll up the injection flows, and thus the mixing process between the fuel and the freestream is accelerated.

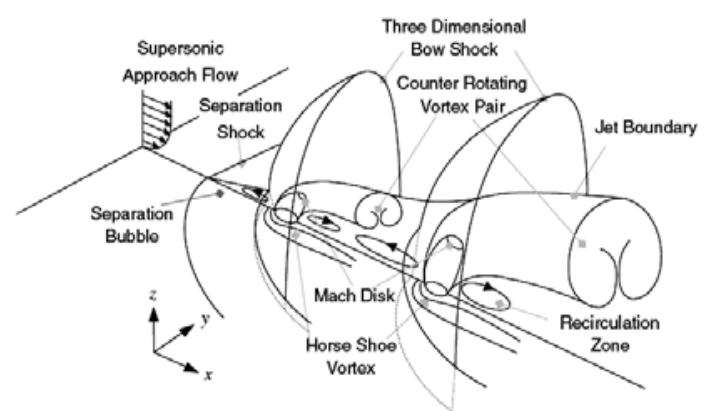

Fig. 9 Schematic view of the mean flow field of the dual transverse injection scheme (Lee, 2006a)

The transverse injection flow field through multiple injection ports in a Mach 4.0 crossflow was computed by Pudsey and Boyce (2010). They found that the selection of the turbulence model and the wall boundary conditions has a great impact on the refined flow field structure that is obtained. The total pressure recovery ability is improved due to the decrease of the intensity of the bow shock waves generated in the multi-port injection flow field, as shown in Fig. 10 
(Pudsey and Boyce, 2010), and the total pressure recovery efficiency increases with the increase of the number of injection ports (Fig. 11) (Pudsey and Boyce, 2010). In Fig. 10, the bow shock waves for each case were presented as well. The number of wake vortices in the region behind each injector exit increases with the increase of the number of jets, and the occurrence of vortex structures acts to increase total pressure loss. In Fig. 11, 1J, 4J, 8J, and 16J refer to the injection port number, SA and SST are two turbulence models, and STW and WF are two

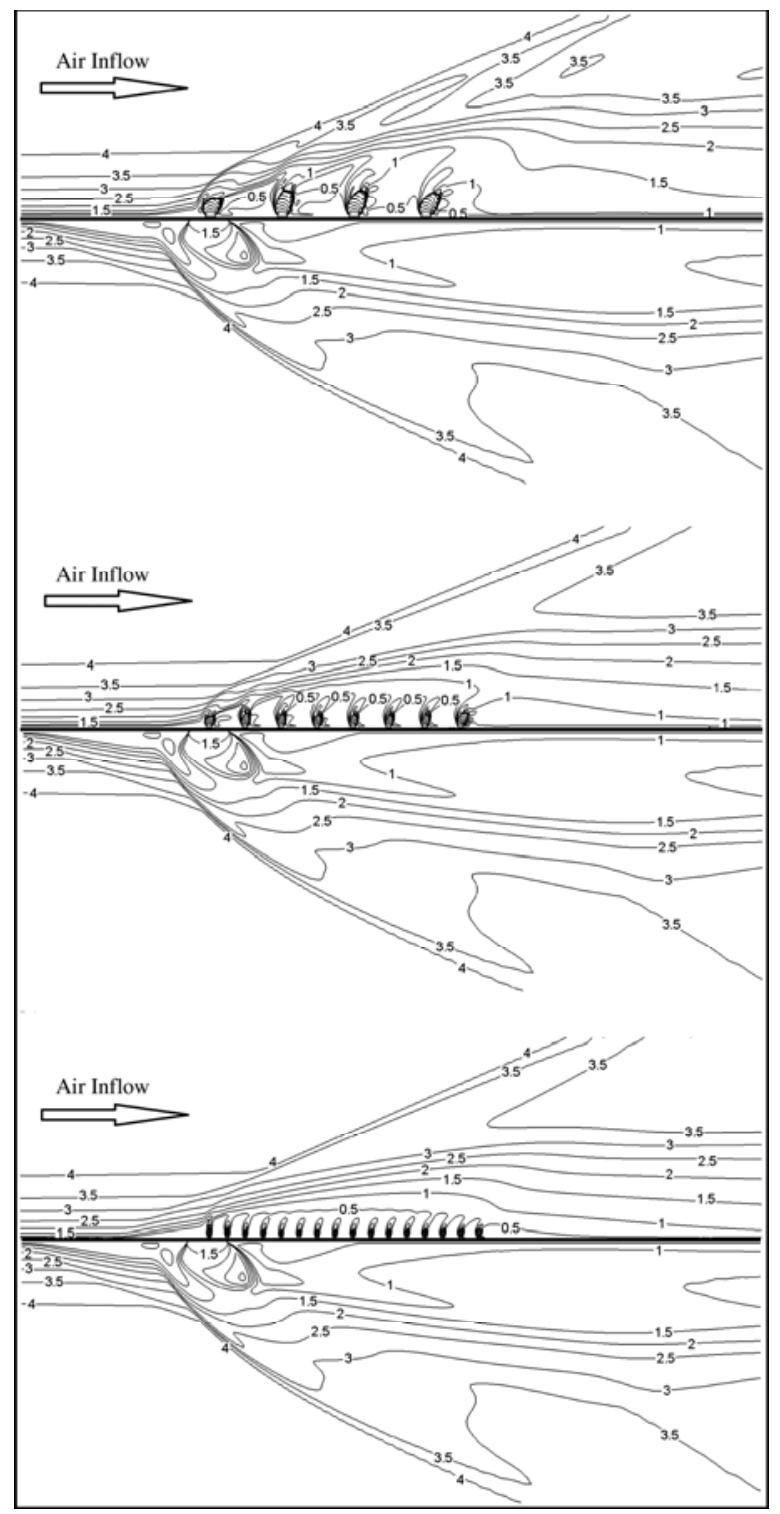

Fig. 10 Mach number contour comparisons of multi-jet cases (upper half of each plot) to single-jet case (lower half) (Pudsey and Boyce, 2010) different wall treatments. $P_{0}$ and $P_{0, \infty}$ are the mass weighted total pressure and its respective freestream total pressure, respectively. At the same time, the fuel penetration depth decreases with the decrease of the injection port diameter and the increase of the injection port number, and there exists an optimal number of injection ports to promote mixing performance between the fuel and the freestream, as shown in Fig. 12 (Pudsey and Boyce, 2010).

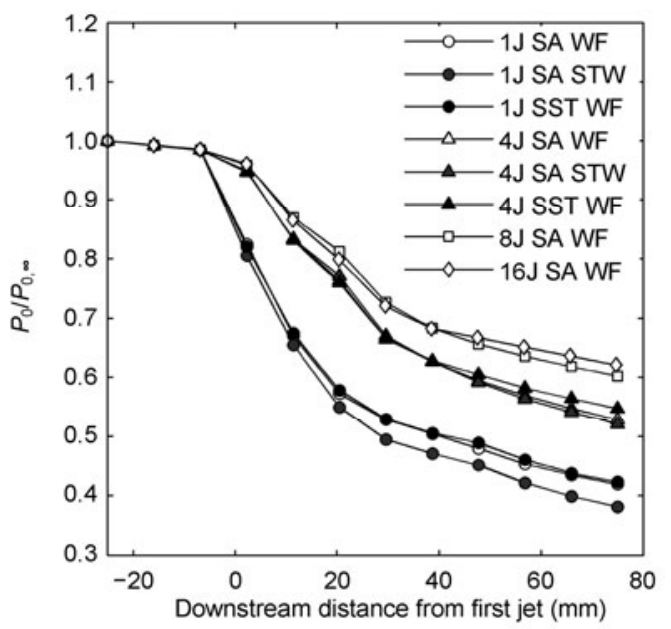

Fig. 11 Total pressure recovery efficiency for different injection schemes (Pudsey and Boyce, 2010)

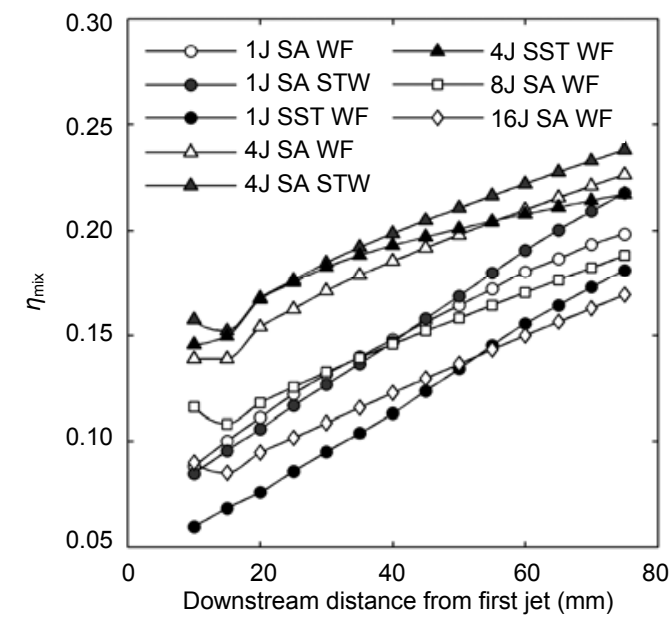

Fig. 12 Comparison for the mixing efficiencies of multi-jet cases (Pudsey and Boyce, 2010)

The dual transverse injection scheme can obtain higher mixing efficiency compared with the single injection scheme when the mass flow rate of the fuel is kept constant; however, the dual transverse 
injection scheme would induce a slightly larger total pressure loss (Gao and Lee, 2011).

The planar laser-induced fluorescence technique was employed by Takahashi et al. (2010a) to investigate the advantages of the dual injection scheme on penetration depth and mixing enhancement, and they found that the dual injection scheme can increase the fuel penetration depth efficiently, but it has no significant advantage in mixing. Lee (2006a; 2006b) analyzed the mixing and combustion flow fields of the dual injection scheme numerically. The influences of the jet-to-crossflow momentum flux ratio and the distance between the injection ports on the mixing and combustion performances were discussed. In the mixing flow field, the jet-to-crossflow momentum flux ratio and the distance between the injection ports both have a large impact on the mixing efficiency, and there exists an optimal distance for the mixing enhancement between the fuel and the freestream. The optimal distance increases with the increase of the jet-to-crossflow momentum flux ratio. In the combustion flow field, the dual injection scheme can improve the combustion efficiency and the flame height compared with the single injection scheme; however, the dual injection scheme would induce larger total pressure loss, and there exists an optimal distance to improve the combustion performance of the dual injection flow field as well. The optimal distance also increases with the increase of the jet-to-crossflow momentum flux ratio.

At the same time, the combination of the dual transverse injection scheme and the backward-facing step was widely employed in the supersonic flowpath (Abbitt et al., 1993; Chakraborty et al., 2003; Manna and Chakraborty, 2005) to enhance the mixing process between the fuel and the supersonic freestream. The combustion efficiency of the scramjet engine was improved thereby.

\section{Effect of injection angle}

The numerical simulation approach was employed by Abdelhafez et al. (2007) to compare the relative performance of oblique and transverse injections in a scramjet combustor. They found that the oblique injection scheme gives higher mixing efficiency and effectiveness compared with the trans- verse injection scheme, which is due to the oblique injection scheme making use of the interaction of the injection-induced shock waves with the air/fuel shear layer. At the same time, the oblique injection scheme can provide better performance in respect of total pressure loss, flow blockage, and boundary layer separation. However, the selection of the injection angle has a strong relationship with the incoming boundary condition and the geometric configuration, and the equivalence ratio is one of the most important control variables for the mixing length. Therefore, this flow field must be optimized by using the multi-objective design optimization approach. Abdelhafez et al. (2007) concluded that the optimal injection angle is $5^{\circ}$, and it can minimize the total pressure loss and improve the mixing efficiency between the fuel and the supersonic freestream. The thrust performance of the engine can thus be improved. In this section, the case with the injection angle being $90^{\circ}$ is the normal injection scheme.

The effect of injection angle with the circularshaped injection port on the mixing flow field in the Mach 3.8 freestream was studied experimentally and numerically by Aso et al. (2009). They found that the larger injection angle can induce a larger total pressure loss, and mixing efficiency is promoted rapidly in the recirculation zone when the injection angle is $150^{\circ}$. In this condition, the case with the injection angle of $150^{\circ}$ is a counter flow injection.

Ali and Sadrul Islam (2006) used a numerical simulation approach to investigate the influences of freestream angle and injection angle on the interaction process between the freestream and the injection flow. It was concluded that mixing of the fuel and the freestream is dominated by the recirculation zone upstream of the injection port; however, it is dominated by the mass fraction of the fuel downstream of the injection port. Meanwhile, the traverse injection scheme can obtain the largest mixing efficiency, and its large recirculation zone upstream of the injection port has a significant flame-holding function.

The Schlieren method was employed by Yang et al. (2012) to study the combustion trajectory in a transverse injection flow field. The quantitative relationship between the fuel penetration depth, the injection angle, and the injection pressure was generated using vector analysis, namely 
$\frac{y}{d}=1.702\left(\mathrm{e}^{\cos \alpha}\right)^{-0.117}(\sin \alpha)^{1.807}\left(\frac{p_{2}-p_{1}}{p_{0}-p_{1}}\right)^{0.553}\left(\frac{x}{d}\right)^{0.296}$,

where $p_{2}, p_{1}$, and $p_{0}$ represent the total pressure of the freestream, the static pressure of the freestream, and the power of the high pressure nitrogen, respectively. $d$ and $\alpha$ are the injector diameter and the injection angle, respectively.

Gao and Lee (2011) found that the variation of the injection angle under circular-shaped injection mainly affects the near-field mixing degree, and the case with $120^{\circ}$ injection angle shows the highest mixing efficiency in the range they considered, as shown in Fig. 13 (Gao and Lee, 2011). In this condition, there is a counter flow injection case as well. This implies that the optimal injection angle has a strong relationship with the freestream boundary condition and the geometric configuration of the injection port, and each design variable should be considered. Thus, the optimization of the transverse injection flow field is very important and urgent.

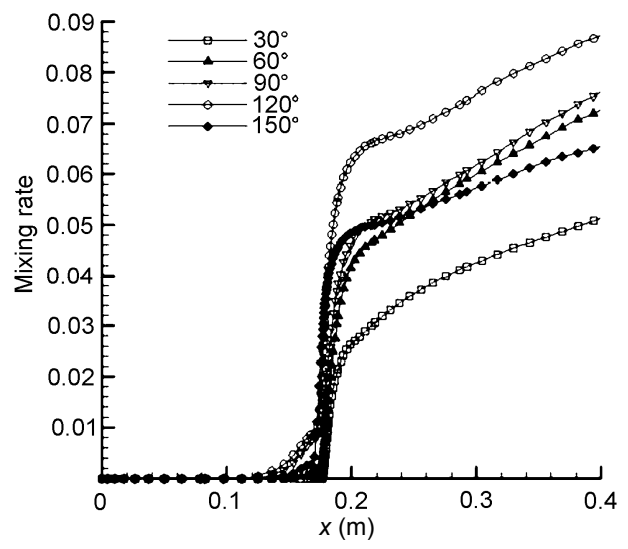

Fig. 13 Comparison of mixing efficiencies along $x$-axis for different injection angles (Gao and Lee, 2011)

\section{Conclusions}

In this survey, research on the mixing techniques of the transverse injection flow field has been summarized from four aspects: the jet-to-crossflow pressure ratio, the geometric configuration of the injection port, the number of injection ports, and the injection angle. The following conclusions can be made as follows.

1. To obtain a refined structure for the transverse injection flow field, various numerical simulation approaches with high fidelity were used, from RANS to large eddy simulation (LES), and then from LES to detached eddy simulation (DNS) (Peterson and Candler, 2010; Ilak et al., 2011; Zhang et al., 2011; Hassan et al., 2013). Their main purpose is to explore the mixing mechanism in the transverse injection flow field. This is important for the design of the fuel injection strategy in scramjet engines.

2. To promote the mixing process between the fuel and the supersonic freestream, a combination of the transverse injection scheme and any other flame holder was widely employed in the supersonic flowpath, i.e., backward-facing step, strut (Zou et al., 2007; Mura and Izard, 2010; Huang et al., 2011b), ramp. This is the main candidate for future approaches to fuel injection, and to improvement in the overall performance of scramjet engines.

3. The staged injection scheme can promote the mixing process between the fuel and the freestream in the supersonic flow more efficiently than the single injection scheme. However, the staged injection scheme inevitably induces larger total pressure loss. At the same time, the diamond-shaped injection port can promote mixing between the fuel and the freestream better than the circular-shaped injection port when the jet-to-crossflow momentum flux ratio is low; the refined flow field structure with high jet-to-crossflow momentum flux ratio needs to be explored further.

4. The design of the transverse injection flow field includes many design variables and objective functions, and it is a multi-objective multi-variable problem. Moreover, there must be a compromise among the objective functions. Thus, the quantitative indexes of this flow field should be optimized by using the multi-objective design optimization approach, and then those optimized results that lie in the Pareto front should be dealt with and visualized using data mining theory (Huang et al., 2013b). This would provide more intuitive design ideas for researchers so they can select the optimal configuration for engineering application from the best choices obtained by the multi-objective design optimization approach. 


\section{References}

Abbitt, J.D.III, Segal, C., McDaniel, J.C., Krauss, R.H., Whitehurst, R.B., 1993. Experimental supersonic hydrogen combustion employing staged injection behind a rearward-facing step. Journal of Propulsion and Power, 9(3):472-478. [doi:10.2514/3.23646]

Abdelhafez, A., Gupta, A.K., Balar, R., Yu, K.H., 2007. Evaluation of Oblique and Traverse Fuel Injection in a Supersonic Combustor. AIAA Paper 2007-5026.

Ali, M., Sadrul Islam, A.K.M., 2006. Study on main flow and fuel injector configurations for scramjet applications. International Journal of Heat and Mass Transfer, 49(19-20):3634-3644. [doi:10.1016/j.ijheatmasstransfer. 2005.12.023]

Aso, S., Okuyama, S., Kawai, M., Ando, Y., 1991. Experimental Study on Mixing Phenomena in Supersonic Flows with Slot Injection. AIAA Paper 91-0016.

Aso, S., Inoue, K., Yamaguchi, K., Tani, Y., 2009. A study on supersonic mixing by circular nozzle with various injection angles for air breathing engine. Acta Astronautica, 65(5-6):687-695. [doi:10.1016/j.actaastro.2009.01.051]

Beresh, S.J., Henfling, J.F., Erven, R.J., Spillers, R.W., 2006. Crossplane velocimetry of a transverse supersonic jet in a transonic crossflow. AIAA Journal, 44(12):3051-3061. [doi:10.2514/1.22311]

Bowersox, R.D.W., Fan, H., Lee, D., 2004. Sonic injection into a Mach 5.0 freestream through diamond orifices. Journal of Propulsion and Power, 20(2):280-287. [doi:10.2514/ 1.9254]

Brieschenk, S., O’Byrne, S., Kleine, H., 2013. Laser-induced plasma ignition studies in a model scramjet engine. Combustion and Flame, 160(1):145-148. [doi:10.1016/j. combustflame.2012.08.011]

Cecere, D., Ingenito, A., Giacomazzi, E., Romagnosi, L., Bruno, C., 2011. Hydrogen/air supersonic combustion for future hypersonic vehicles. International Journal of Hydrogen Energy, 36(18):11969-11984. [doi:10.1016/j. ijhydene.2011.06.051]

Chakraborty, D., Roychowdhury, A.P., Ashok, V., Kumar, P., 2003. Numerical investigation of staged transverse sonic injection in Mach 2 stream in confined environment. The Aeronautical Journal, 107:719-729.

Chen, L.W., Wang, G.L., Lu, X.Y., 2011. Numerical investigation of a jet from a blunt body opposing a supersonic flow. Journal of Fluid Mechanics, 684:85-110. [doi:10. 1017/jfm.2011.276]

Feng, X.P., Lin, Z.Y., Zheng, Y., Li, J.X., Cao, Q., 2011. Adjusting principle of gas jet controlling inlet and numerical verification. Science in China Series E: Technological Sciences, 54(11):2981-2986. [doi:10.1007/s11431-0114553-3]

Gao, Z.X., Lee, C.H., 2011. Numerical research on mixing characteristics of different injection schemes for supersonic transverse jet. Science in China Series E: Technological Sciences, 54(4):883-893. [doi:10.1007/s11431010-4277-9]
Guoskov, O.V., Kopchenov, V.I., Lomkov, K.E., Vinogradov, V.A., Waltrup, P.J., 2001. Numerical research of gaseous fuel preinjection in hypersonic three-dimensional inlet. Journal of Propulsion and Power, 17(6):1162-1169. [doi:10.2514/2.5890]

Hassan, E., Boles, J., Aono, H., Davis, D., Shyy, W., 2013. Supersonic jet and crossflow interaction: Computational modeling. Progress in Aerospace Sciences, 57:1-24, [doi:10.1016/j.paerosci.2012.06.002]

Huang, W., Qin, H., Luo, S.B., Wang, Z.G., 2010. Research status of key techniques for shock-induced combustion ramjet (scramjet) engine. Science in China Series E: Technological Sciences, 53(1):220-226. [doi:10.1007/ s11431-009-0379-7]

Huang, W., Pourkashanian, M., Wang, Z.G., Ma, L., Ingham, D.B., Luo, S.B., Liu, J., Xia, Z.X., Lei, J., Jin, L., Wang, Z.W., 2011a. Overview of Fuel Injection Techniques for Scramjet Engines. Proceedings of ASME Turbo Expo, British Columbia, Canada.

Huang, W., Wang, Z.G., Luo, S.B., Liu, J., 2011b. Parametric effects on the combustion flow field of a typical strutbased scramjet combustor. Chinese Science Bulletin, 56(35):3871-3877.

Huang, W., Liu, W.D., Li, S.B., Xia, Z.X., Liu, J., Wang, Z.G., 2012a. Influences of the turbulence model and the slot width on the transverse slot injection flow field in supersonic flows. Acta Astronautica, 73:1-9. [doi:10.1016/j. actaastro.2011.12.003]

Huang, W., Ma, L., Pourkashanian, M., Ingham, D.B., Luo, S.B., Wang, Z.G., 2012b. Parametric effects in a scramjet engine on the interaction between the air stream and the injection. Proceedings of the Institution of Mechanical Engineers, Part G: Journal of Aerospace Engineering, 226(3):294-309. [doi:10.1177/0954410011408512]

Huang, W., Wang, Z.G., Wu, J.P., Li, S.B., 2013a. Numerical prediction on the interaction between the incident shock wave and the transverse slot injection in supersonic flows. Aerospace Science and Technology, 28(1):91-99. [doi:10. 1016/j.ast.2012.10.007]

Huang, W., Wang, Z.G., Ingham, D.B., Ma, L., Pourkashanian, M., 2013b. Design exploration for a single expansion ramp nozzle (SERN) using data mining. Acta Astronautica, 83:10-17. [doi:10.1016/j.actaastro.2012.09.016]

Huang, W., Li, S.B., Yan, L., Wang, Z.G., 2013c. Performance evaluation and parametric analysis on cantilevered ramp injector in supersonic flows. Acta Astronautica, 84:141152. [doi:10.1016/j.actaastro.2012.11.011]

Ilak, M., Schlatter, P., Bagheri, S., Chevalier, M., Henningson, D.S., 2011. Stability of a jet in crossflow. Physics of Fluids, 23(9):091113. [doi:10.1063/1.3640011]

Kobayashi, K., Bowersox, R.D.W., Srinivasan, R., Tichenor, N.R., Carter, C.D., Ryan, M.D., 2010. Experimental and numerical studies of diamond-shaped injector in a supersonic flow. Journal of Propulsion and Power, 26(2): 373-376. [doi:10.2514/1.47147] 
Kovar, A., Schulein, E., 2006. Comparison of experimental and numerical investigation on a jet in a supersonic cross-flow. The Aeronautical Journal, 110:353-360.

Lee, S.H., 2006a. Characteristics of dual transverse injection in scramjet combustor, Part 1: Mixing. Journal of Propulsion and Power, 22(5):1012-1019. [doi:10.2514/1.14180]

Lee, S.H., 2006b. Characteristics of dual transverse injection in scramjet combustor, Part 2: Combustion. Journal of Propulsion and Power, 22(5):1020-1026. [doi:10.2514/1. 14185]

Li, Z.W., Huai, W.X., Qian, Z.D., 2012. Study on the flow field and concentration characteristics of the multiple tandem jets in crossflow. Science in China Series E: Technological Sciences, 55(10):2778-2788. [doi:10.1007/s11431012-4964-9]

Livingston, T., Segal, C., Schindler, M., Vinogradov, V.A., 2000. Penetration and spreading of liquid jets in an external-internal compression inlet. AIAA Journal, 38(6): 989-994. [doi: 10.2514/2.1082]

Manna, P., Chakraborty, D., 2005. Numerical investigation of transverse sonic injection in a non-reaction supersonic combustor. Proceedings of the Institution of Mechanical Engineers, Part G: Journal of Aerospace Engineering, 219(2):205-215. [doi:10.1243/095440805X8601]

Mura, A., Izard, J.F., 2010. Numerical simulation of supersonic nonpremixed turbulent combustion in a scramjet combustor model. Journal of Propulsion and Power, 26(4): 858-868. [doi:10.2514/1.48074]

Ogawa, H., Boyce, R.R., 2012. Multi-objective Design Optimization of Fuel Injection for Mixing Enhancement in Scramjets by Using Surrogate-assisted Evolutionary Algorithms. 18th AIAA/3AF International Space Planes and Hypersonic Systems and Technologies Conference, AIAA Paper 2012-5815.

Peterson, D.M., Candler, G.V., 2010. Hybrid Reynoldsaveraged and large-eddy simulation of normal injection into a supersonic crossflow. Journal of Propulsion and Power, 26(3):533-544. [doi:10.2514/1.46810]

Pudsey, A.S., Boyce, R.R., 2010. Numerical investigation of transverse jets through multiport injector arrays in supersonic crossflow. Journal of Propulsion and Power, 26(6): 1225-1236. [doi:10.2514/1.39603]

Pudsey, A.S., Boyce, R.R., Wheatley, V., 2012. Hypersonic Viscous Drag Reduction via Multi-Porthole Injector Arrays. 18th AIAA/3AF International Space Planes and Hypersonic Systems and Technologies Conference, Tours, France, AIAA Paper 2012-5934.

Qin, J., Bao, W., Zhang, S.L., Song, Y.F., Yu, D.R., 2012. Thermodynamic analysis for a chemically recuperated scramjet. Science in China Series E: Technological Sciences, 55(11):3204-3212. [doi:10.1007/s11431-0125000-9]

Rizzetta, D., 1992. Numerical Simulation of Slot Injection into a Turbulent Supersonic Stream. 30th Aerospace Sciences Meeting \& Exhibit, Reno, NV, AIAA Paper 92-0827.
Seiner, J.M., Dash, S.M., Kenzakowski, D.C., 2001. Historical survey on enhanced mixing in scramjet engines. Journal of Propulsion and Power, 17(6):1273-1286. [doi:10.2514/ 2.5876]

Srinivasan, R., Bowersox, R.D.W., 2008. Transverse injection through diamond and circular ports into a Mach 5.0 freestream. AIAA Journal, 46(8):1944-1962. [doi:10.2514/ 1.29253]

Takahashi, H., Ikegami, S., Masuya, G., Hirota, M., 2010a. Extended quantitative fluorescence imaging for multicomponent and staged injection into supersonic crossflows. Journal of Propulsion and Power, 26(4):798-807. [doi:10.2514/1.47318]

Takahashi, H., Tu, Q., Segal, C., 2010b. Effects of pylon-aided fuel injection on mixing in a supersonic flowfield. Journal of Propulsion and Power, 26(5):1092-1101. [doi:10.2514/ 1.48393]

Tomioka, S., Kohchi, T., Masumoto, R., Izumikawa, M., Matsuo, A., 2011. Supersonic combustion with supersonic injection through diamond-shaped orifices. Journal of Propulsion and Power, 27(6):1196-1203. [doi:10.2514/ 1.B34164]

Turner, J.C., Smart, M.K., 2010. Application of inlet injection to a three-dimensional scramjet at Mach 8. AIAA Journal, 48(4):829-838. [doi:10.2514/1.J050052]

Wang, H.B., Qin, N., Sun, M.B., Wu, H.Y., Wang, Z.G., 2011a. A hybrid LES (Large Eddy Simulation)/assumed sub-grid PDF (Probability Density Function) model for supersonic turbulent combustion. Science in China Series E: Technological Sciences, 54(10):2694-2707. [doi:10. 1007/s11431-011- 4518-6]

Wang, X.W., Cai, G.B., Gao, Y.S., Huo, H.F., 2011b. High flowrate injector with gaseous hydrogen and gaseous oxygen. Science in China Series E: Technological Sciences, 54(11):2958-2973. [doi:10.1007/s11431-0114538-2]

Wang, Y.W., Sislian, J.P., 2010. Numerical simulation of gaseous hydrocarbon fuel injection in a hypersonic inlet. Journal of Propulsion and Power, 26(5):1114-1124. [doi:10.2514/1.47741]

Xu, S.L., Archer, R.D., Milton, B.E., Yue, P.T., 2000. Unsteady transverse injection of kerosene into a supersonic flow. Science in China Series E: Technological Sciences, 43(2):206-214. [doi:10.1007/BF02916892]

Yang, H., Li, F., Sun, B., 2012. Trajectory analysis of fuel injection into supersonic cross flow. Chinese Journal of Aeronautics, 25(1):42-50. [doi:10.1016/S1000-9361(11) 60360-9]

Yang, H.Q., Kim, T.B., Lu, T.J., 2011. Characteristics of annular impinging jets with/without swirling flow by short guide vanes. Science in China Series E: Technological Sciences, 54(3):749-757. [doi:10.1007/s11431010-4233-8]

You, Y.C., Ludeke, H., Hannemann, K., 2012. On the flow physics of a low momentum flux ratio jet in a supersonic turbulent crossflow. 97:24001. [doi:10.1209/0295-5075/ 97/24001] 
You, Y.C., Luedeke, H., Hannemann, K., 2013. Injection and Mixing in a Scramjet Combustor: DES and RANS Studies. Proceedings of the Combustion Institute, 34(2): 2083-2092. [doi:10.1016/j.proci.2012.10.001]

Zhang, H.Q., Rong, Y., Wang, B., Wang, X.L., 2011. Large eddy simulation of a 3-D spatially developing turbulent round jet. Science in China Series E: Technological Sciences, 54(11):2916-2923. [doi:10.1007/s11431-0114577-8]

Zhang, H.Q., Ga, Y.J., Wang, B., Wang, X.L., 2012. Analysis of combustion instability via constant volume combustion in a LOX/RP-1 bipropellant liquid rocket engine. Science in China Series E: Technological Sciences, 55(4):10661077. [doi:10. 1007/s11431-012-4743-7]

Zhang, P.F., Dai, C.F., Liu, A.B., Wang, J.J., 2011. The effect of actuation frequency on the plasma synthetic jet. Science in China Series E: Technological Sciences, 54(11): 2945-2950. [doi:10.1007/s11431-011-4546-2]

Zou, J.F., Zheng, Y., Liu, O.Z., 2007. Simulation of turbulent combustion in DLR scramjet. Journal of Zhejiang University-SCIENCE A, 8(7):1053-1058. [doi:10.1631/ jzus.2007.A1053]

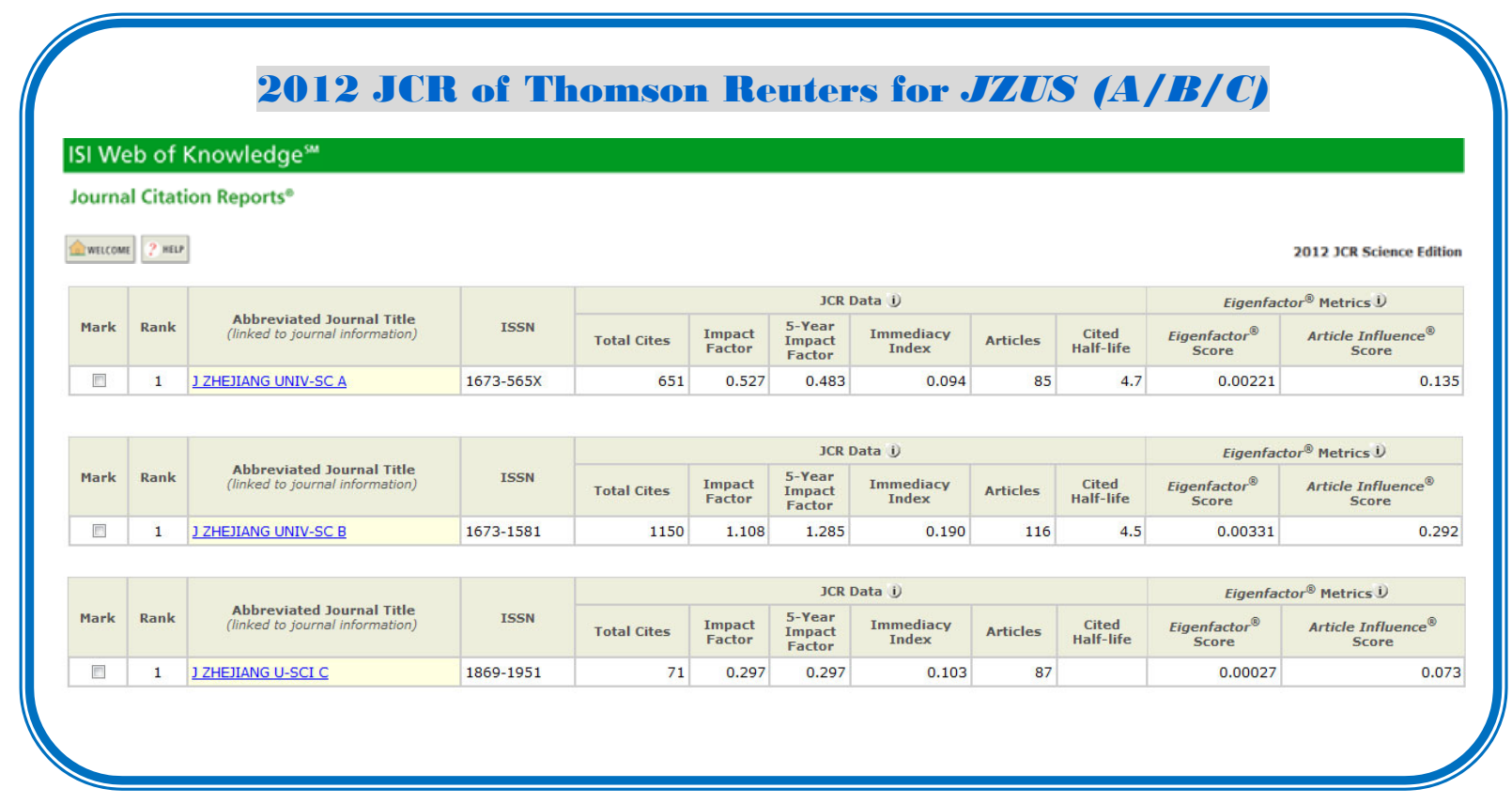

